\title{
Angiolymphoid hyperplasia with eosinophilia attached to the masseter muscle
}

\author{
Hyo Jeong Kwon, \\ Ee Room Jung, \\ Jong Yun Choi, \\ Bommie Florence Seo, \\ Ho Kwon, \\ Sung-No Jung \\ Department of Plastic and \\ Reconstructive Surgery, College of \\ Medicine, The Catholic University of \\ Korea, Seoul, Korea
}

\begin{abstract}
Angiolymphoid hyperplasia with eosinophilia (ALHE) is a rare benign vascular tumor. The pathogenesis of ALHE is unknown; however, it may be linked to local trauma. ALHE predominantly occurs in areas of the preauricular region, forehead, and scalp; the masseter area is rarely involved. A 49-year-old man was referred for a mass in the right cheek region that was felt 2 months prior. Physical and imaging examination results suggested the presence of a benign tumor. Thus, surgical excision was performed. Pathologic findings confirmed an unexpected diagnosis of ALHE. This case was interesting, since the mass occurred at an unusual site with a misdiagnosis of an epidermal inclusion cyst.
\end{abstract}

Keywords: Aangiolymphoid hyperplasia with eosinophilia / Immunohistochemistry / Masseter muscle

\section{INTRODUCTION}

Angiolymphoid hyperplasia with eosinophilia (ALHE) is a rare benign vascular tumor. The pathophysiology of ALHE is unknown, and it is unclear whether it arises from a primary vascular origin, lymphoproliferative process, or heterogeneously. There are many causes of ALHE, including trauma, infection, and hormonal factors [1].

ALHE appears as isolated or grouped papules, and it is often asymptomatic. It may also be accompanied by pain or pruritus. ALHE predominantly occurs in the periauricular region, forehead, and scalp areas. However, the presence of ALHE at other locations has also been reported [2]. It is often confused with other masses; indeed, ALHE is not typically diagnosed at the initial visit because the disease is very rare and its symptoms are usually nonspecific. However, recurrence and incomplete reso-

\footnotetext{
Correspondence: Sung-No Jung

Department of Plastic and Reconstructive Surgery, Uijeongbu St. Mary's Hospital, College of Medicine, The Catholic University of Korea, 271 Cheonbo-ro, Uijeongbu 11765 , Korea

E-mail: jsn7190@catholic.ac.kr

Received July 16, 2020 / Revised August 18, 2020 / Accepted October 19, 2020
}

lution of ALHE are common. Thus, accurate diagnosis of this tumor is crucial, since it can progress toward a chronic course even after complete excision, unlike other benign tumors. We treated a case of ALHE that occurred in the form of a subcutaneous nodule in an unusual location abutting the masseter muscle. In this report, we describe our case and the literature review that was performed.

\section{CASE REPORT}

A 49-year-old man visited the plastic surgery department following palpation of a solitary nodule in the right cheek area (Fig. 1). The patient found this lesion spontaneously 2 months prior to presentation, with no prior history of a similar lesion. The mass was slightly increased in size. However, he had no other symptoms, such as pain or pruritus. On physical examination, a round mass in the right cheek was identified. The mass was approximately $1.5 \times 1.5 \mathrm{~cm}$ in size and was soft-totouch; also, the overlying skin appeared normal. There was no pulsation on the mass. Laboratory tests were performed preoperatively. All values, including serum eosinophils, were within 


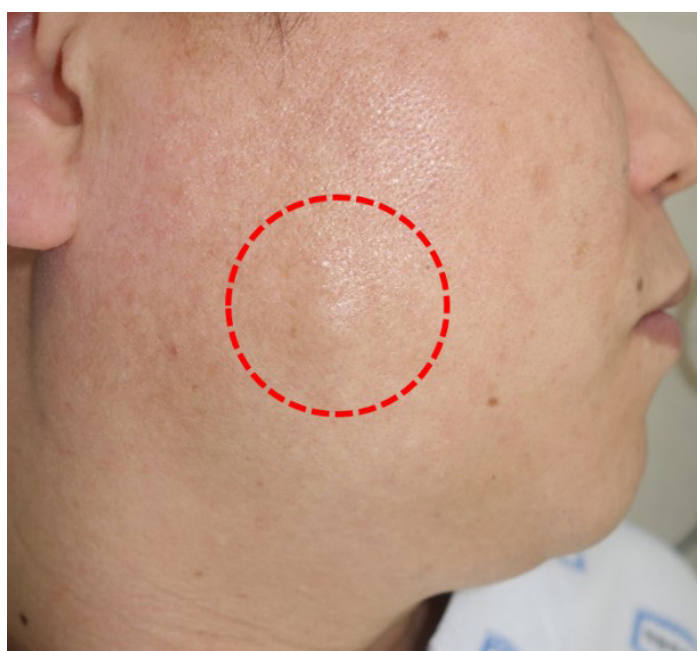

Fig. 1. Preoperative clinical image of the right cheek mass (red circle).

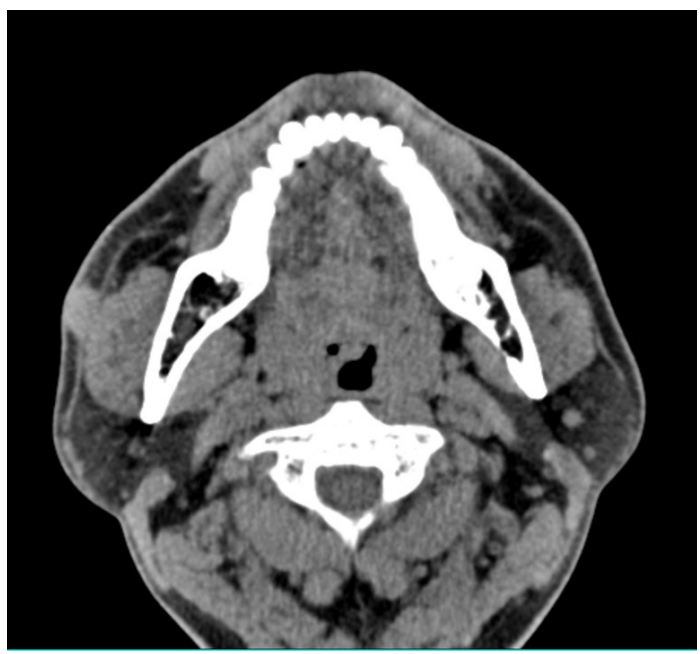

Fig. 2. An enhanced mass abutting the right masseter muscle with a size of approximately $1.5 \times 1.5 \mathrm{~cm}$.

normal limits. In computed tomography, an oval well-defined hyperdensity lesion with a size of approximately $1.5 \mathrm{~cm}$ and mild enhancement was observed in the right buccal subcutaneous tissue (Fig. 2). Based on the above findings, the initial impression was an epidermal inclusion cyst. Thus, surgical excision was performed.

At the time of surgery, the mass was found to be attached to the fascia of the masseter muscle. Total excision of the mass was performed with care not to cause injury to the masseter muscle (Fig. 3). In addition, the mass was removed meticulously, since the location of the mass and the buccal branch of the facial nerve were adjacent. The mass was then sent for histopathological analysis.

Microscopic analysis showed abnormal vascular proliferation and diffuse lymphocytic infiltration with eosinophilia (Fig. 4).

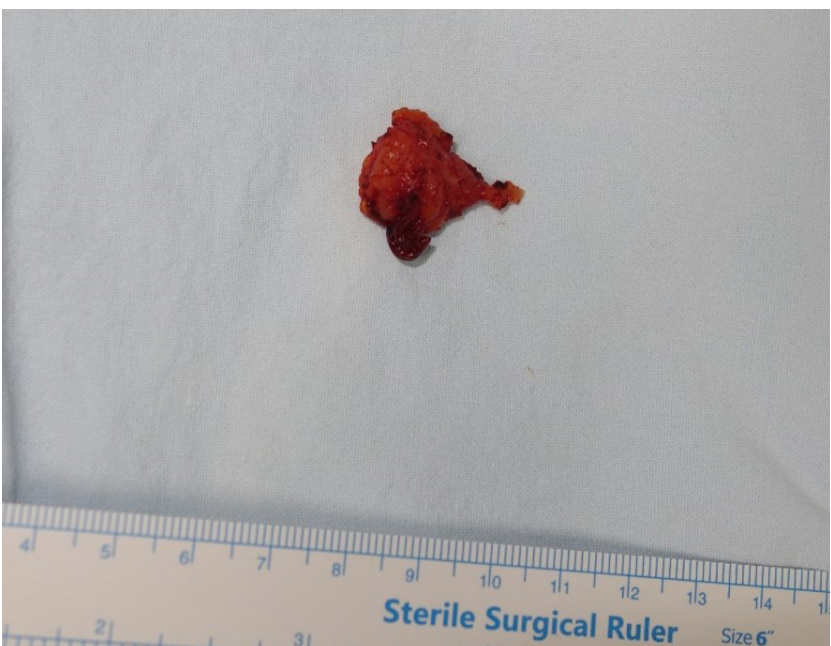

Fig. 3. Pinkish specimen on the right masseter muscle.

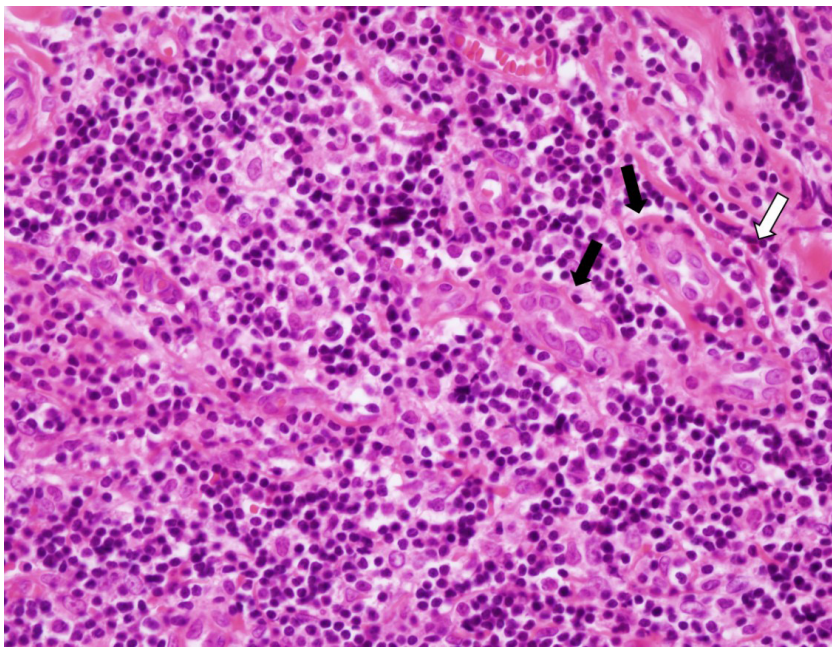

Fig. 4. Photomicrograph showing enlarged blood vessels due to endothelial hypertrophies (black arrows) and eosinophilia (white arrow) (H\&E, $\times 400)$.

Immunohistochemistry was performed to confirm the diagnosis, assuming that it was a type of atypical lymphoproliferative disease, not an epidermal inclusion cyst. Staining was positive for $\mathrm{CD} 3$ and $\mathrm{CD} 20$ (Fig. 5). CD3 is a specific marker in the surrounding mantle zone of T-lymphocytes, and CD20 is a specific marker in the germinal center of B-lymphocytes. The final diagnosis was ALHE based on the above findings. The surgical site was well maintained, and there was no recurrence at a 1 -year follow-up.

\section{DISCUSSION}

ALHE, also known as epithelioid hemangioma, is a very rare benign vascular tumor. It was first described by Wells and Whimster in 1969 [3]; however, it remains unclear how ALHE 

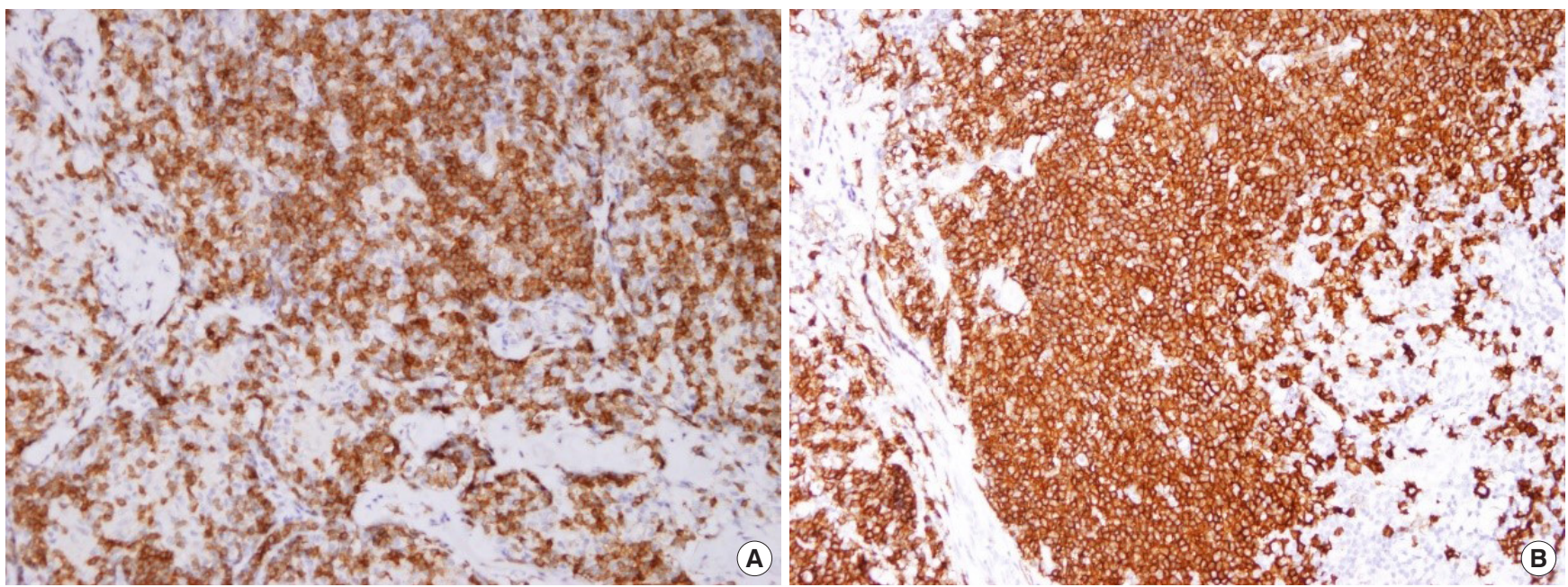

Fig. 5. Immunohistochemistry showing CD3 $(A, \times 200)$ and $C D 20(B, \times 200)$ positive regions.

occurs. One hypothesis is that it is associated with an arteriovenous shunt from advanced trauma, that is, vascular neoplasia with rich inflammatory components [4]. A second hypothesis is that it occurs as a result of the lymphoproliferative process. Additionally, it might also be linked to hormonal causes, such as a hyperestrogenic state. It is unclear whether ALHE is primarily of vascular origin, a lymphoproliferative process, or heterogeneous [5-7]. However, most cases of ALHE presented in the literature resulted from local trauma. In the present case, the patient recognized the mass spontaneously without a history of associated trauma. We hypothesize that in this case ALHE may have been generated due to irritation from constant movement of the masseter due to the location of the lesion.

ALHE may be asymptomatic, and it is sometimes accompanied by pain, bleeding, and pruritus [8]. The most common location of ALHE is the periauricular region, forehead, and scalp. It can also occur in many other areas, such as the trunk, lower extremities, and oral mucosa. To the best of our knowledge, there have been no reports of ALHE abutting to the masseter muscle.

In most cases, ALHE appears as a single or multiple nodules from a few millimeters to several centimeters in diameter. Occasionally, it is accompanied by lesions of the overlying skin. As in our case, it is often confused with an epidermal inclusion cyst. It could be difficult to differentiate from pyogenic granuloma or keloid if there is a history of trauma; thus, caution is required in these cases. It is especially crucial to distinguish ALHE from Kimura's disease as a differential diagnosis. ALHE and Kimura's disease were considered the same line of disease in the past [9]. However, many studies have recently agreed that these two are separate diseases with different entities. Histopathologically, ALHE is characterized by enlarged blood vessels of various sizes and endothelial cell hypertrophy accompanied by increased eosinophils. Kimura's disease mainly affects lymphoid follicles without invading blood vessels. Thus, the main difference between ALHE and Kimura's disease is that ALHE is of vascular origin, while Kimura's disease is a chronic inflammatory process that occurs mainly in young Asian males [10]. Additionally, regional lymphadenopathy is very rare in ALHE. Peripheral blood eosinophilia occurs in $10 \%$ to $20 \%$ of cases. The immunoglobulin E rate was almost normal in ALHE. However, in Kimura's disease, lymphadenopathy and peripheral eosinophilia are common, and immunoglobulin E levels are increased. Based on these differences, it is possible to differentiate between these two diseases.

The treatment of choice for ALHE is surgical excision. This is due to the fact that ALHE is mainly expressed in mass form. Also, pathological confirmation is required. Corticosteroids, imiquimod, laser, local radiotherapy, and cryotherapy can also be used for treatment of this disease, with satisfactory results. Although ALHE benign, recurrence, and incomplete resolution are common. Indeed, accurate diagnosis is crucial since it can progress toward a chronic course even after complete excision, unlike other benign tumors. According to the literature, there have been no cases of malignancy. However, patients should be informed of the possibility of chronicity and recurrence. Thus, they should be regularly monitored through outpatient follow-up.

In the case presented herein, we initially believed that the lesion was an epidermal inclusion cyst of the cheek. However, we later discovered that it was ALHE, a relatively rare disease with pathologic confirmation. ALHE is not a well-known disease; also, it appears in various clinical forms. Therefore, typically it is not initially suspected. However, its diagnosis is possible based on its histological features. The knowledge about ALHE 
provided in this case report will enable surgeons to consider ALHE for a patient with a palpable mass at any site.

\section{NOTES}

\section{Conflict of interest}

No potential conflict of interest relevant to this article was reported.

\section{Ethical approval}

The study was approved by the Institutional Review Board of Uijeongbu St. Mary's Hospital (IRB No. UC20ZASI0071) and performed in accordance with the principles of the Declaration of Helsinki. Written informed consent was obtained.

\section{Patient consent}

The patient provided written informed consent for the publication and the use of his images.

\section{ORCID}

Hyo Jeong Kwon https://orcid.org/0000-0002-5778-5692 Ee Room Jung https://orcid.org/0000-0001-6510-8045 Jong Yun Choi https://orcid.org/0000-0002-1164-4499 Bommie Florence Seo https://orcid.org/0000-0002-6907-5962 Ho Kwon https://orcid.org/0000-0001-7471-0804 Sung-No Jung https://orcid.org/0000-0002-0419-4717

\section{REFERENCES}

1. Bahloul E, Amouri M, Charfi S, Boudawara O, Mnif H, Boudawara T, et al. Angiolymphoid hyperplasia with eosinophilia: report of nine cases. Int J Dermatol 2017;56:1373-8.

2. Tambe SA, Nayak CS. Successful management of angiolymphoid hyperplasia with eosinophilia by radiofrequency. J Cu$\tan$ Aesthet Surg 2017;10:116-8.

3. Wells GC, Whimster IW. Subcutaneous angiolymphoid hyperplasia with eosinophilia. Br J Dermatol 1969;81:1-14.

4. Olsen TG, Helwig EB. Angiolymphoid hyperplasia with eosinophilia: a clinicopathologic study of 116 patients. J Am Acad Dermatol 1985;12(5 Pt 1):781-96.

5. Okman JS, Bhatti TR, Jackson OA, Rubin AI. Angiolymphoid hyperplasia with eosinophilia: a previously unreported complication of ear piercing. Pediatr Dermatol 2014;31:738-41.

6. Vadlamudi G, Schinella R. Traumatic pseudoaneurysm: a possible early lesion in the spectrum of epithelioid hemangioma/ angiolymphoid hyperplasia with eosinophilia. Am J Dermatopathol 1998;20:113-7.

7. Busquets AC, Sanchez JL. Angiolymphoid hyperplasia with eosinophilia induced by trauma. Int J Dermatol 2006;45:1211-4.

8. Adler BL, Krausz AE, Minuti A, Silverberg JI, Lev-Tov H. Epidemiology and treatment of angiolymphoid hyperplasia with eosinophilia (ALHE): a systematic review. J Am Acad Dermatol 2016;74:506-12.

9. Chong WS, Thomas A, Goh CL. Kimura's disease and angiolymphoid hyperplasia with eosinophilia: two disease entities in the same patient: case report and review of the literature. Int J Dermatol 2006;45:139-45.

10. Fite-Trepat L, Martos-Fernandez M, Alberola-Ferranti M, Pablo-Garcia-Cuenca A, Bescosatin C. Angiolymphoid hyperplasia with eosinophilia involving the occipital artery: case report and review of literature. J Clin Diagn Res 2017;11:ZD21-3. 